\title{
Efficacy and safety of combined immunosuppressive therapy plus umbilical cord blood infusion in severe aplastic anemia patients: A cohort study
}

\author{
XIU LUO*, HUINA LU*, BING XIU, HAO WU, BING LI, PING LI, YUHUA CHEN, \\ LILI ZHOU, WENJUN ZHANG, YAN DONG, AIBIN LIANG and YI DING \\ Department of Hematology, Tongji Hospital, Tongji University School of Medicine, Shanghai 200065, P.R. China
}

Received May 19, 2017; Accepted August 22, 2017

DOI: $10.3892 / \mathrm{etm} .2017 .5616$

\begin{abstract}
The present study aimed to evaluate the efficacy and safety of combined immunosuppressive therapy (IST) plus umbilical cord blood infusion (UCBI) in severe aplastic anemia (SAA) patients. A total of 68 patients with SAA were enrolled in the current prospective cohort study and divided into the IST $(n=35$; positive control) and IST+UCBI $(n=33$; experimental) groups according to the treatment conditions. Patients in the IST group were treated with rabbit antithymocyte globulin (r-ATG) at a dose of $2.5 \mathrm{mg} / \mathrm{kg}$ through intravenous infusion once a day for five days. This was combined with oral cyclosporine A (CsA) at a dose of $3-5 \mathrm{mg} / \mathrm{kg}$ twice a day for 2 years. Patients in the IST+UBCI group were treated with r-ATG and CsA at the same doses and frequencies as the IST group plus one UCBI 1 day after the final treatment with r-ATG. At 6 months post treatment, the complete response and overall response rate (ORR) of the IST+UCBI group were markedly higher compared with those in the IST group. Furthermore, patients in the IST+UCBI group achieved absolute neutrophil count (ANC) and platelet count responses more rapidly as compared with the IST group. However, no difference in the hemoglobin $(\mathrm{Hb})$ response was
\end{abstract}

Correspondence to: Dr Yi Ding or Dr Aibin Liang, Department of Hematology, Tongji Hospital, Tongji University School of Medicine, 389 Xincun Road, Shanghai 200065, P.R. China

E-mail: 18621908870@163.com

E-mail: lab7182@tongji.edu.cn

Abbreviations: IST, immunosuppressive therapy; UCBI, umbilical cord blood infusion; AA, aplastic anemia; SAA, severe aplastic anemia; VSAA, very severe aplastic anemia; ORR, overall response rate; $\mathrm{Hb}$, hemoglobin; ANC, absolute neutrophil count; HSCT, hematopoietic stem-cell transplantation; ATG, antithymocyte globulin; CsA, cyclosporine A; HPCs, hematopoietic progenitor cells

*Contributed equally

Key words: efficacy, immunosuppressive therapy, umbilical cord blood infusion, severe aplastic anemia, very severe aplastic anemia identified between the two groups. In addition, SAA patients achieved responses in the ANC and platelet count more rapidly in comparison with very severe aplastic anemia (VSAA) patients, while the number of days to $\mathrm{Hb}$ responses were similar in the SAA and VSAA patients. Multivariate logistic regression analysis also revealed that IST+UCBI treatment was an independent predicting factor for patients achieving complete response or partial response, whereas VSAA was an independent predictor of a worse ORR. Platelet and reticulocyte were also independent predicting factors. Finally, the survival of patients was similar between the groups, and no difference in the safety of the treatment was observed. In conclusion, combined IST plus UCBI treatment may be applied as an effective and safe therapy for SAA patients.

\section{Introduction}

Aplastic anemia (AA) is a complex hematologic disease, during which the hematopoietic progenitor and stem cells (HSPCs) are reduced due to attack by autoreactive cytotoxic T cells, which eventually leads to pancytopenia (1). Severe AA (SAA) is an aggressive type of AA that remains problematic due to its poor prognosis. Generally, patients can be treated with hematopoietic stem-cell transplantation (HSCT); however, allogeneic human leukocyte antigen (HLA)-matched sibling donors are rare for the majority of patients (2). Many previous studies have focused on identifying the methods of pathogenesis associated with disoriented immune function, including type 1 cytotoxic T cell (Tc1) dysfunction, which is the immune disorder associated with the etiology of AA (3-7). The disorented function of cytotoxic $\mathrm{T}$ cells in patients with AA may result in the HSPCs being targeted by cytotoxic $\mathrm{T}$ cells, consequently the HSPCs will be largely diminished. Thus, due to the good efficacy observed by earlier studies, immunosuppressive therapy (IST) is considered as the main conditioning regimen in clinical practice for patients lack sibling donors or not suitable for $\operatorname{HSCT}(8,9)$. Although a good therapeutic response to IST that involves a combination of antithymocyte globulin (ATG) and cyclosporine A (CsA) has been observed in AA and SAA patients, the appearance of refractory and relapse SAA remains relatively frequent, indicating a need for an improved treatment strategy (1,10-13). 
Umbilical cord blood infusion (UCBI) is becoming increasingly popular in the treatment of autoimmune disease, such as type 1 diabetes (14-16). During UCBI treatment, the potential curative effect of UCB partially results from the presence of immature unprimed highly-functional $\mathrm{T}$ cells, which are able to inhibit the inflammatory cytokines in immune disorders $(17,18)$. UCB also contains hematopoietic cells, the expansion of which restores the peripheral blood cells to a certain extent (19). Furthermore, a previous study observed a good survival rate of SAA patients treated with unrelated UCB transplantation, with a median survival of 750 days (20). However, combination therapy of IST plus UCBI in SAA patients has not been well investigated or applied in clinical practice to date.

Thus, the aim of the present study was to evaluate the efficacy and safety of combined IST plus UCBI in the treatment of SAA patients.

\section{Materials and methods}

Study participants. In total, 68 patients diagnosed with SAA at the Department of Hematology in Tongji Hospital (Shanghai, China) were enrolled in the present prospective cohort study between March 2009 and February 2012. The inclusion criteria were as follows: i) Age, $>2$ years; ii) weight, $>12 \mathrm{~kg}$; iii) diagnosis of SAA according to the Camitta criteria $(21,22)$, which was defined as a bone marrow cellularity of $<30 \%$ (excluding lymphocytes) and at least two of the following factors: Absolute neutrophil count (ANC) of $<0.5 \times 10^{9}$ cells $/ 1$, platelet count of $<20 \times 10^{9}$ cells $/ 1$ and absolute reticulocyte count of $<20 \times 10^{9}$ cells $/ 1$. The exclusion criteria in the present study were the following: i) Diagnosis of Fanconi anemia (23); ii) patients with clinically evident congestive heart failure, serious cardiac arrhythmias, or symptoms of coronary artery disease; iii) pregnant or breastfeeding women; iv) patients with medical, psychological or social conditions that may interfere with their participation in the study; and v) positive human immunodeficiency virus (HIV) test. The current study was approved by the local Ethics Committee of Tongji Hospital and conducted according to the Declaration of Helsinki (24). Written informed consent was obtained from all patients recruited to the present study.

Definitions. Very severe AA (VSAA) was defined as an ANC of $<0.2 \times 10^{9} / 1$ in patients diagnosed with SAA (25). The overall response rate (ORR) was defined as the proportion of patients who achieved complete response (CR) and partial response (PR). An ANC response was defined as an ANC of $>0.5 \times 10^{9} / 1$, a platelet response was categorized as a platelet count of $>20 \times 10^{9} / 1$ and a hemoglobin $(\mathrm{Hb})$ response was defined as an $\mathrm{Hb}$ of $>80 \mathrm{~g} / \mathrm{l}$.

Treatment. The treatments administered to patients were not disrupted their inclusion within the present study. All therapeutic strategies were conducted by qualified physicians according to clinical guidelines. Patients were divided into the IST (control group; 23 males, 12 females; aged 8-52 years) and the IST+UCBI (experimental group; 19 males, 14 females; aged 12-50 years) groups according to treatment they were administered. In the IST group, patients were treated with rabbit antithymocyte globulin (r-ATG; Genzyme Polyclonals S.A.S.; Sanofi Genzyme, Cambridge, MA, USA) at a dose of $2.5 \mathrm{mg} / \mathrm{kg}$ by intravenous infusion once a day for 5 days. This was combined with oral cyclosporine A (CsA; Huadong Medicine Co., Ltd., Hangzhou, China) at a dose of $3-5 \mathrm{mg} / \mathrm{kg}$ twice a day for a minimum of 2 years (maintaining the trough level at $150-250 \mu \mathrm{g} / \mathrm{l})$. In the IST+UBCI group, patients were treated with r-ATG and CsA at the same doses and frequencies as patients in the IST group, plus UCBI, which was conducted only once on each patient 1 day after the final r-ATG treatment. All the UCB samples used in the present study were obtained from the Shanghai Cord Blood Bank (Shanghai, China). Tests were performed on the UCB by a qualified researcher to screen for hepatitis B virus, HIV, blood type and other routine tests prior to and following collection. UCB was subsequently transfused into the patients.

In addition to the IST and UCBI treatments, support therapy was provided to patients. To minimize the risk of infection, all patients slept in laminar flow beds prior to the initiation of combined IST. These beds enable air filtration, which reduces the prevalence of fungus and bacteria surrounding the patient. Prior to ATG treatment, $0.5-1.0 \mathrm{mg} / \mathrm{kg}$ promethazine was injected intramuscularly into the patients. During therapy glucocorticoids were used to prevent allergic reactions, and patients were carefully monitored to ensure that any cases of serum sickness were treated rapidly. Furthermore, condensed erythrocyte infusion was performed when the $\mathrm{Hb}$ level was $<60 \mathrm{~g} / 1$, and platelet infusion was conducted on patients when the platelet count was $<10 \times 10^{9} / 1$ or $>10 \times 10^{9} / 1$ combined with the occurrence of bleeding or symptoms of high risk of intracranial hemorrhage. Additionally, patients were treated with granulocyte colony-stimulating factor (Beijing Four Rings Biopharmaceutical Co., Ltd., Beijing, China) when their ANC was $<1.5 \times 10^{9} / 1$.

Assessment. The efficacy of treatment in the current study was evaluated according to the International Camitta Criteria $(21,22)$ as follows: i) CR was defined as an ANC of $>1.5 \times 10^{9} / 1, \mathrm{Hb}$ level $>110 \mathrm{~g} / \mathrm{l}$ and platelet count $\geq 150 \times 10^{9} / 1$; ii) PR was defined as an ANC of $>0.5 \times 10^{9} / 1, \mathrm{Hb}$ of $>80 \mathrm{~g} / \mathrm{l}$ and platelet count $>20 \times 10^{9} / 1$, with no transfusion dependence for platelets or red blood cells; iii) relapse was determined when the peripheral blood count decreased to a level of requiring transfusions or reinstitution of IST (or HSCT) (26). CR, PR and relapse were assessed at 6 months post treatment. In addition, the peripheral hemogram, liver function, renal function and blood glucose of patients were evaluated. Furthermore, the degree of HLA match was tested using a serum assay prior to treatment with UCBI. In the serum assay the HLA-DR antigens-associated invariant chain (cat. no, AF3590; R\&D Systems, Inc., Minneapolis, MN, USA) was used for the ELISA assay, which was performed at room temperature according to the manufacturer's protocol. Briefly, the HLA-DR antigen was diluted into solution with protein at a concentration of $1-10 \mu \mathrm{g} / \mathrm{ml}$ using a carbonate coating buffer, subsequently $0.1 \mathrm{ml}$ of the solution was added into each well of the polyethylene plate, which was incubated overnight at $4^{\circ} \mathrm{C}$. The following day the plate was washed in $\mathrm{pH}$ 7.4, PBS 3 times ( 3 min each time). The samples were then diluted and added into each well $\left(\begin{array}{lll}0 & 1 \mathrm{ml}\end{array}\right)$ and the plate was incubated for $1 \mathrm{~h}$ at $37^{\circ} \mathrm{C}$ and then washed again with PBS as described above. The CD74 
Table I. Baseline characteristics of patients in the IST and IST+UCBI groups.

\begin{tabular}{|c|c|c|c|}
\hline Parameters & IST group $(\mathrm{n}=35)$ & IST+UCBI group $(n=33)$ & P-value \\
\hline Age, years & $29.88 \pm 12.84$ & $29.71 \pm 16.79$ & 0.964 \\
\hline Gender (male/female), $\mathrm{n}$ & $23 / 12$ & $19 / 14$ & 0.497 \\
\hline BMI, $\mathrm{kg} / \mathrm{m}^{2}$ & $21.54 \pm 1.94$ & $22.28 \pm 2.10$ & 0.134 \\
\hline Diagnosis to IST, months & $32.0(20.0-51.0)$ & $61.0(37.5-133.5)$ & $<0.001$ \\
\hline Etiology, n (\%) & & & 0.953 \\
\hline Idiopathic & $33(94.3)$ & $31(93.9)$ & \\
\hline Other & $2(5.7)$ & $2(6.1)$ & \\
\hline Severity of disease, $\mathrm{n}(\%)$ & & & 0.68 \\
\hline VSAA & $8(22.9)$ & $9(27.3)$ & \\
\hline SAA & $27(77.1)$ & $24(72.7)$ & \\
\hline ANC, $\times 10^{9}$ cells $/ 1$ & $0.310(0.202-0.444)$ & $0.295(0.194-0.407)$ & 0.641 \\
\hline Platelets, $\times 10^{9}$ cells/ 1 & $15.924(10.288-23.471)$ & $12.661(5.809-18.758)$ & 0.101 \\
\hline Reticulocytes, $\times 10^{9}$ cells $/ 1$ & $18.454(11.152-26.062)$ & $17.881(11.651-27.436)$ & 0.773 \\
\hline Hemoglobin, $\mathrm{g} / \mathrm{l}$ & $59.523(48.315-77.988)$ & $66.653(50.349-80.934)$ & 0.308 \\
\hline HLA match, n (\%) & & & - \\
\hline $4 / 6$ & - & $11(33)$ & \\
\hline $5 / 6$ & - & $11(33)$ & \\
\hline $6 / 6$ & - & $11(33)$ & \\
\hline Cell dose & & & - \\
\hline $\operatorname{TNC}\left(10^{7} / \mathrm{kg}\right)$ & - & $2.412 \pm 0.846$ & \\
\hline $\mathrm{CD}_{3} 4^{+}\left(10^{5} / \mathrm{kg}\right)$ & - & $0.585 \pm 0.33$ & \\
\hline
\end{tabular}

Data are mainly expressed as the mean \pm standard division, count (percentage) or median (25th-75th percentile). Comparison between two groups was detected by Student's t-test, Wilcoxon rank-sum test or $\chi^{2}$ test. IST, immunosuppressive therapy; UCBI, umbilical cord blood infusion; BMI, body mass index; VSAA, very severe aplastic anemia; SAA, severe aplastic anemia; ANC, absolute neutrophil count; HLA, human leukocyte antigen; TNC, total nuclear cell.

antibody (dilution 1-10 $\mu \mathrm{m} / \mathrm{ml}$; cat. no. orb396383; Biorbyt Ltd., Cambridge, UK) labeled with horseradish peroxidase was diluted with PBS containing $1.0 \%$ bull serum albumin (cat. no. 10099141; Thermo Fisher Scientific, Inc., Waltham, MA, USA) and $0.1 \mathrm{ml}$ was added into each well and incubated for 0.5 to $1 \mathrm{~h}$ at $37^{\circ} \mathrm{C}$, followed by washing with PBS. Following this, $0.1 \mathrm{ml}$ tetramethylbenzidine substrate solution was added to each well and incubated for $10-30 \mathrm{~min}$ at $37^{\circ} \mathrm{C}$. A total of $0.05 \mathrm{ml} 2 \mathrm{M}$ sulfuric acid solution was added to terminate the reaction. The final results were read on a microplate reader (Bio-Rad Laboratories, Inc., Hercules, CA, USA), and the optical density (OD) value was assessed under $450 \mathrm{~nm}$ following setting the machine to zero using the blank well. If the OD value was 1.2 fold different to the value of the blank well, the result was considered positive. The reference level was $2.5-7.5 \times 10^{9} / 1$ for ANC and 100-300 $\times 10^{9} / 1$ for platelets. The reference levels of $\mathrm{Hb}$ for males and females were 120-160 g/l and 110-150 g/l, respectively. The concentration of CsA was monitored in order to readjust the dose as required. The overall survival (OS) of patients was recorded at 5 years after the initiation of the study.

Statistical analysis. SPSS version 21.0 software (IBM Corp., Armonk, NY, USA) was used for statistical analysis. Data are presented as the mean \pm standard division, count (percentage) or median (25-75th percentile). Comparisons between groups were determined by Student t-test, $\chi^{2}$ test or Wilcoxon rank-sum test. Kaplan-Meier curve analysis and the log-rank test were performed to evaluate the response time and OS of patients between the groups. Logistic regression analysis was also conducted to assess the factors predicting the ORR. $\mathrm{P}<0.05$ was considered to indicate a difference that was statistically significant.

\section{Results}

Baseline characteristics of patients in the IST and IST+UCBI groups. As listed in Table I, the mean age of patients was $29.88 \pm 12.84$ years in the IST group and $29.71 \pm 16.79$ years in the IST+UCBI group ( $\mathrm{P}=0.964)$. There were 23 males and 12 females in the IST group, while 19 males and 14 females were included in the IST+UCBI group $(\mathrm{P}=0.497)$. The body mass index of patients was $21.54 \pm 1.94 \mathrm{~kg} / \mathrm{m}^{2}$ in the IST group and $22.28 \pm 2.10 \mathrm{~kg} / \mathrm{m}^{2}$ in the IST+UCBI group $(\mathrm{P}=0.134)$. The interval between diagnosis and the initiation of IST in patients in the IST+UCBI group was notably longer compared with that in the IST group $(\mathrm{P}<0.001)$. No statistically significant difference in the baseline demographic and other characteristics was detected between the two groups (Table I). Additionally, 27 patients were categorized as SAA and 8 patients were diagnosed with VSAA in the IST group, while there were 24 SAA 
Table II. Clinical response of IST and IST+UCBI treatment after 6 months.

\begin{tabular}{lccr}
\hline Parameters & IST group $(\mathrm{n}=35)$ & IST+UCBI group $(\mathrm{n}=33)$ & P-value \\
\hline ORR (CR+PR), n (\%) & $20(57.1)$ & $27(81.8)$ & 0.028 \\
CR, n (\%) & $14(40.0)$ & $22(66.7)$ & 0.028 \\
PR, n (\%) & $6(17.1)$ & $5(15.2)$ & 0.824 \\
Relapse, n (\%) & $0(0.0)$ & $1(3.0)$ & 0.299 \\
\hline
\end{tabular}

Comparison between the two groups was conducted by $\chi^{2}$ test. IST, immunosuppressive therapy; UCBI, umbilical cord blood infusion; ORR, overall response rate; $\mathrm{CR}$, complete response; $\mathrm{PR}$, partial response.

Table III. Individual ANC, platelet count and hemoglobin level responses in the IST and IST+UCBI treatment groups after 6 months.

\begin{tabular}{lccc}
\hline Parameters & IST group, $n(\%)$ & IST+UCBI group, n (\%) & P-value \\
\hline ANC response & $29 / 32(90.6)$ & $28 / 28(100.0)$ & 0.096 \\
Platelet response & $12 / 20(60.0)$ & $23 / 28(82.1)$ & 0.089 \\
Hemoglobin response & $19 / 28(67.8)$ & $20 / 25(80.0)$ & 0.551 \\
\hline
\end{tabular}

Comparison between the two groups was conducted by $\chi^{2}$ test. IST, immunosuppressive therapy; UCBI, umbilical cord blood infusion; ANC, absolute neutrophil count.

and 9 VSAA patients in the IST+UBCI group $(\mathrm{P}=0.680)$. Furthermore, the TNC was $2.412 \pm 0.84610^{7} / \mathrm{kg}$ and the $\mathrm{CD} 34^{+}$ was $0.585 \pm 0.3310^{5} / \mathrm{kg}$ in the IST+UCBI group.

Clinical response of patients in the IST and IST+UCBI groups. At 6 months post treatment, the ORR of the IST+UCBI group was markedly higher compared with that of the IST group ( 81.8 vs. $57.1 \%$; $\mathrm{P}=0.028$; Table II). In addition, a greater number of patients achieved CR in the IST+UCBI group (22 patients; 66.7\%) as compared with the IST group (14 patients; $40.0 \% ; \mathrm{P}=0.028$ ). The number of patients that achieved PR in the IST+UCBI and IST groups were $5(15.2 \%)$ and $6(17.1 \%)$, respectively $(\mathrm{P}=0.824)$. Among all patients, only 1 patient $(3 \%)$ relapsed in the IST+UCBI group, while there was no relapse in the IST group ( $\mathrm{P}=0.299$; Table II).

Days to ANC, platelet count and Hb level responses of patients in the IST and IST+UCBI groups. As shown in Table III, 29 patients $(90.6 \%)$ achieved an ANC response out of the 32 patients with $\mathrm{ANC}<0.5 \times 10^{9}$ cells $/ 1$ at baseline in the IST group, whereas all the 28 patients (100.0\%) with $\mathrm{ANC}<0.5 \times 10^{9}$ cell/1 at baseline achieved an ANC response in the IST+UCBI group $(\mathrm{P}=0.096)$. As presented in Fig. 1A, patients achieved an $\mathrm{ANC}$ response more rapidly in the IST+UCBI group compared with the IST group $(\mathrm{P}<0.001)$.

Regarding the platelet concentration, 12 patients $(60 \%)$ achieved a platelet response among the 20 patients in the IST group with a platelet count of $<20 \times 10^{9}$ cells $/ 1$ at baseline (Table III). By contrast, among the 28 patients in the IST+UCBI group with a platelet count of $<20 \times 10^{9}$ cells $/ 1$ at baseline, 23 cases $(82.1 \%)$ achieved a platelet response $(\mathrm{P}=0.089)$. The rate of patients achieving platelet response was significantly faster in the IST+UCBI group, as displayed in Fig. 1B.

In the IST group, $19(67.8 \%)$ of the 28 patients who presented an $\mathrm{Hb}$ level of $<80 \mathrm{~g} / \mathrm{l}$ achieved an $\mathrm{Hb}$ response. Similarly, in the IST+UCBI group, among the 25 patients with $\mathrm{Hb}<80 \mathrm{~g} / \mathrm{dl}, 20$ (67.8\%) patients achieved an $\mathrm{Hb}$ response (Table III). However, there was no marked difference in the number of days to achieve the $\mathrm{Hb}$ response between the IST and IST+UCBI groups ( $\mathrm{P}=0.153$; Fig. 1C).

Days to ANC, platelet count and Hb level responses in VSAA and SAA patients. In terms of the comparison between VSAA and SAA patients, the number of days required to achieve an ANC response in the SAA patients was significantly lower in comparison the VSAA patients, while more SAA patients achieved an ANC response compared with the VSAA patients $(\mathrm{P}=0.045$; Fig. 2A). In addition, a similar result was observed in the number of days to achieve a platelet response between the VSAA and SAA patients ( $\mathrm{P}=0.037$; Fig. $2 \mathrm{~B})$. Regarding the days required to achieve an $\mathrm{Hb}$ response in SAA and VSAA patients, no statistically significant difference was detected $(\mathrm{P}=0.397$; Fig. 2C).

Analysis of baseline factors affecting the ORR. To evaluate the factors affecting the ORR, logistic regression was conducted (Table IV). Univariate logistic regression displayed that, among all baseline factors, IST+UCBI $(\mathrm{P}=0.032)$ treatment increased the possibility of patients achieving CR or PR, while patients with VSAA $(\mathrm{P}=0.006)$ were less likely to achieve $\mathrm{CR}$ or PR. In addition, the platelet count $(\mathrm{P}=0.085)$ and $\mathrm{Hb}$ level $(\mathrm{P}=0.036)$ were also predicting factors of the ORR. All factors with $\mathrm{P}<0.1$ were subsequently 

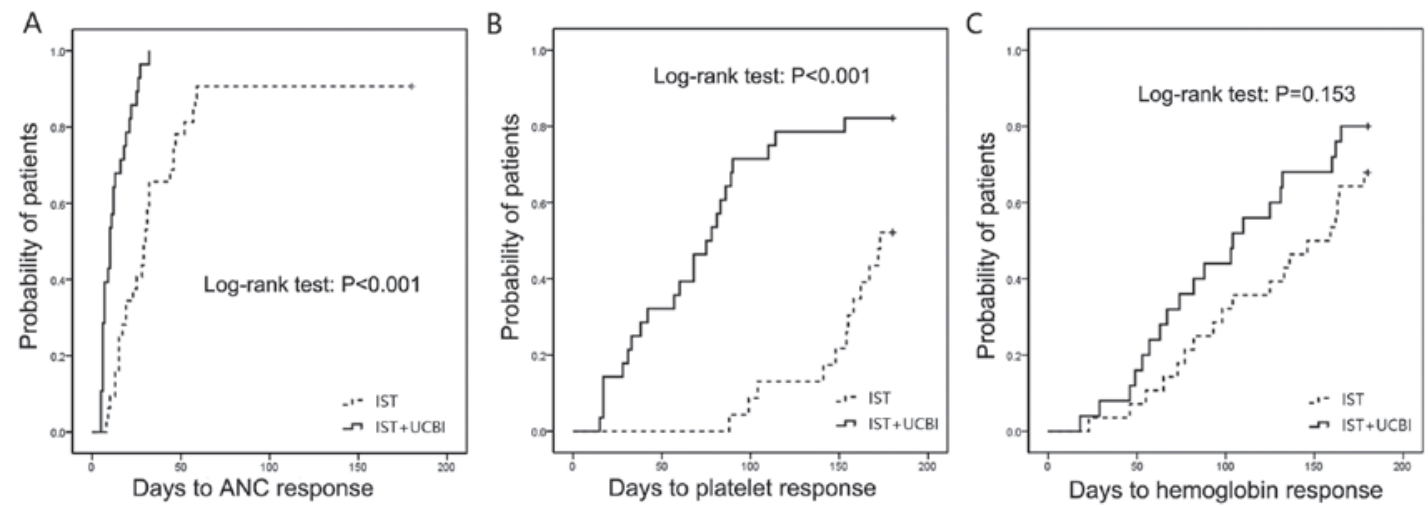

Figure 1. Number of days required to achieve (A) ANC, (B) platelet count and (C) Hb level response in patients of the IST and IST+UCBI groups. A Kaplan-Meier curve analysis and a log-rank test were performed to evaluate the days required to achieve ANC, platelet and Hb responses between the groups. IST, immunosuppressive therapy; UCBI, umbilical cord blood infusion; ANC, absolute neutrophil count; Hb, hemoglobin.
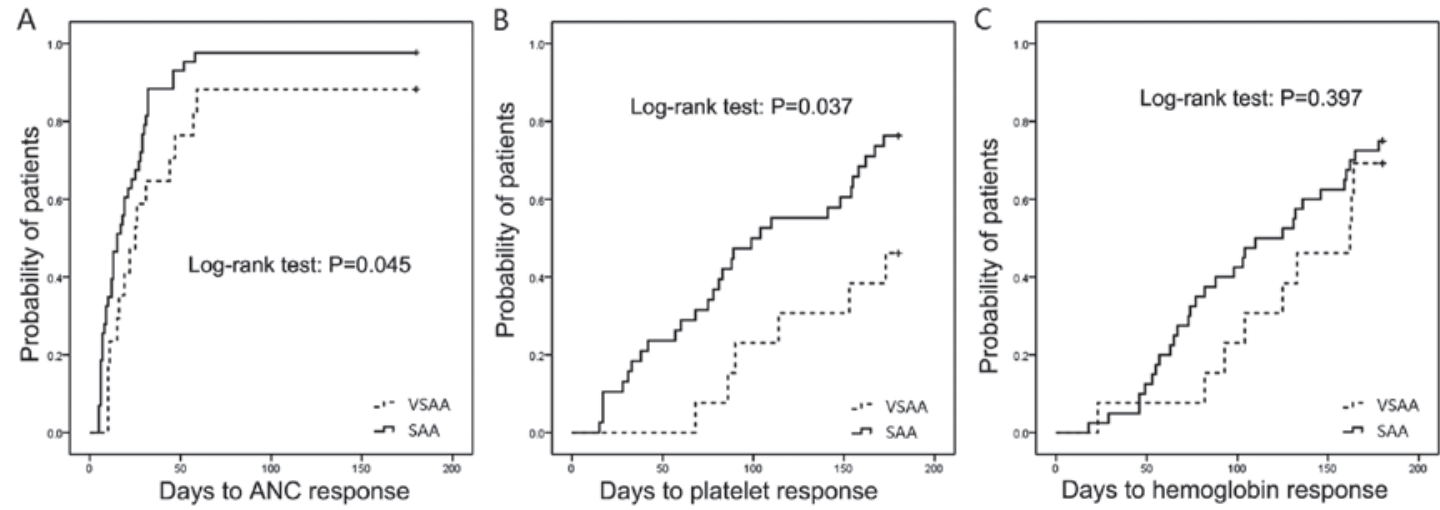

Figure 2. Number of days required to observe an (A) ANC, (B) platelet count and (C) Hb level response in SAA and VSAA patients. A Kaplan-Meier curve analysis and a log-rank test were performed to evaluate the days required to achieve ANC, platelet and $\mathrm{Hb}$ responses between the groups. The treatment responses displayed in the figure are the combined results of the IST and IST+UCBI groups. SAA, severe aplastic anemia; VSAA, very severe aplastic anemia; ANC, absolute neutrophil count; $\mathrm{Hb}$, hemoglobin.

analyzed by multivariate logistic regression, which revealed that IST+UCBI $(\mathrm{P}=0.006)$ was an independent factor for predicting whether patients would achieve CR or PR $(\mathrm{P}=0.006)$. Furthermore, the diagnosis of VSAA independently predicted a worse ORR in patients as compared with SAA $(\mathrm{P}=0.002)$. The platelet $(\mathrm{P}=0.020)$ and reticulocyte $(\mathrm{P}=0.033)$ counts were also independent predicting factors of the ORR, as observed in Table IV.

Survival of patients in the IST and IST+UCBI groups. The 5-year survival rates in the IST and IST+UCBI groups were 82.9 and $93.9 \%$, respectively. As demonstrated in Fig. 3, no significant difference in the OS was identified between the IST and IST+UCBI groups $(\mathrm{P}=0.171)$.

Subgroup analysis in patients with diversified degrees of HLA match. In order to assess the clinical response and OS in patients with different degrees of HLA match, subgroup analysis was performed. As shown in Fig. 4, no difference was detected in the CR rates among the three HLA groups $(\mathrm{P}=0.580)$, while the $\mathrm{CR}$ rates in patients with HLA 6/6, 5/6 and 4/6 were $72.7,72.7$ and $54.5 \%$, respectively. The ORR rates among the three groups were also not significantly different (90.9, 81.8 and $72.7 \%$, respectively; $\mathrm{P}=0.543$; Fig. 4A). Finally, the OS was similar in patients with diversified degrees of HLA match $(\mathrm{P}=0.599$; Fig. 4B).

Safety of IST and IST+UCBI treatments. During or following the treatment, there are several common adverse events, which were recorded in the current study, including fever, rush, ATG-associated serum disease, infection and hemorrhage. As displayed in Table V, no difference of adverse event occurrence between the groups was observed, suggesting that UCBI treatment did not increase the presence of adverse events in the present study.

\section{Discussion}

Given the good efficacy observed in clinical practice, IST is currently the most widely used therapy for AA patients due to its ability to inhibit the imbalanced immune responses $(27,28)$. In contrast to AA, the prognosis of patients with SAA is poor as a result of high mortality caused by severe infections, particularly invasive fungal infections (29). IST involves the use of several immunosuppressive drugs, including ATG, CsA and cyclophosphamide (1). ATG, widely used for the treatment of graft rejection in organ transplantations, is an immune globulin that depletes $\mathrm{T}$ cells, thus resulting in the reduction 
Table IV. Analysis of factors affecting the ORR.

\begin{tabular}{|c|c|c|c|c|c|c|c|c|}
\hline \multirow[b]{3}{*}{ Parameter } & \multicolumn{4}{|c|}{ Univariate logistic regression } & \multicolumn{4}{|c|}{ Multivariate logistic regression } \\
\hline & \multirow[b]{2}{*}{ P-value } & \multirow[b]{2}{*}{ OR } & \multicolumn{2}{|c|}{$95 \% \mathrm{CI}$} & \multirow[b]{2}{*}{ P-value } & \multirow[b]{2}{*}{ OR } & \multicolumn{2}{|c|}{$95 \% \mathrm{CI}$} \\
\hline & & & Lower & Higher & & & Lower & Higher \\
\hline IST+UCBI (vs. IST) & 0.032 & 3.375 & 1.113 & 10.234 & 0.006 & 9.229 & 1.920 & 44.359 \\
\hline VSAA (vs. SAA) & 0.006 & 0.193 & 0.060 & 0.623 & 0.002 & 0.067 & 0.012 & 0.382 \\
\hline Age, years & 0.321 & 0.983 & 0.950 & 1.017 & - & - & - & - \\
\hline Gender (male) & 0.597 & 0.737 & 0.251 & 2.166 & - & - & - & - \\
\hline $\mathrm{BMI}, \mathrm{kg} / \mathrm{m}^{2}$ & 0.515 & 0.919 & 0.712 & 1.185 & - & - & - & - \\
\hline Diagnosis to IST, months & 0.128 & 1.010 & 0.997 & 1.024 & - & - & - & - \\
\hline Etiology (idiopathic) & 0.794 & 1.364 & 0.133 & 13.933 & - & - & - & - \\
\hline ANC, $x 10^{9}$ cells $/ 1$ & 0.394 & 2.675 & 0.275 & 25.986 & - & - & - & - \\
\hline Platelets, $\mathrm{x} 10^{9}$ cells/ 1 & 0.085 & 1.060 & 0.992 & 1.134 & 0.020 & 1.140 & 1.021 & 1.273 \\
\hline Reticulocytes, $\times 10^{9}$ cells $/ 1$ & 0.632 & 0.993 & 0.963 & 1.023 & - & - & - & - \\
\hline Hemoglobin, g/l & 0.036 & 1.032 & 1.002 & 1.062 & 0.033 & 1.042 & 1.003 & 1.081 \\
\hline
\end{tabular}

Data were analyzed by univariate and multivariate logistic regression analyses. Factors with a $\mathrm{P}<0.1$ in the univariate model were subsequently analyzed by the multivariate model. $\mathrm{P}<0.05$ was considered to indicate statistically significant differences. OR, odds ratio; $95 \% \mathrm{CI}, 95 \%$ confidence interval; IST, immunosuppressive therapy; UCBI, umbilical cord blood infusion; VSAA, very severe aplastic anemia; SAA, severe aplastic anemia; BMI, body mass index; ANC, absolute neutrophil count.

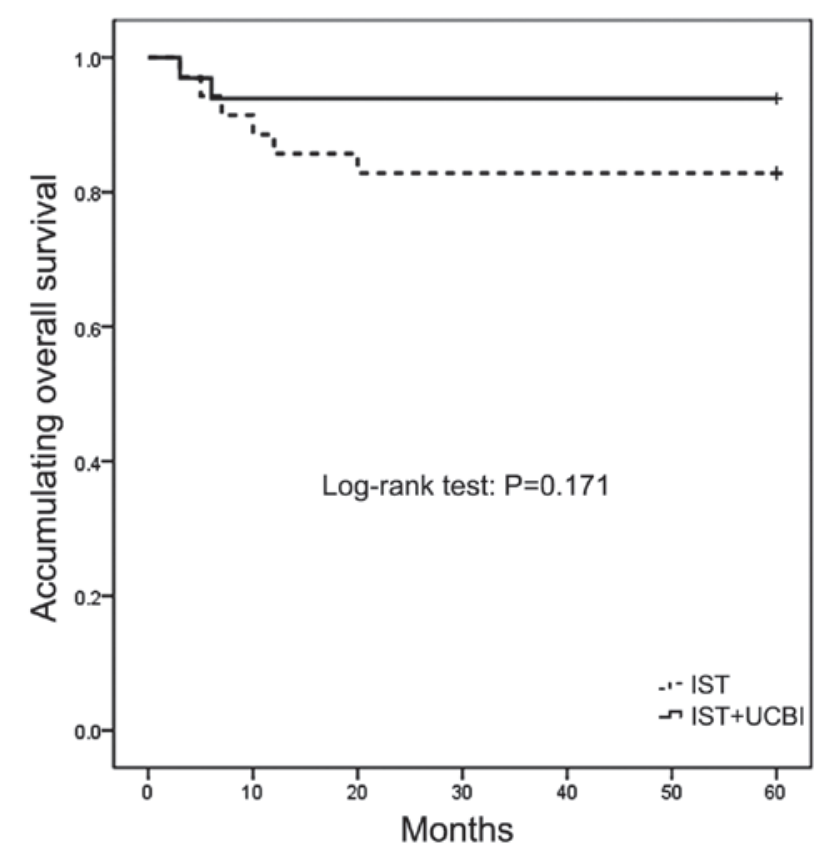

Figure 3. Overall survival of patients in the IST and IST+UCBI group. A Kaplan-Meier curve analysis and a log-rank test were performed to evaluate the overall survival between groups. IST, immunosuppressive therapy; UCBI, umbilical cord blood infusion.

of over activated $\mathrm{T}$ cells and their abnormal activities $(1,30)$. Similarly, CsA is characterized by its ability to alleviate $\mathrm{T}$ cell proliferation and activation, although it presents several toxicities; however, the side effects can be ameliorated by dose readjustment (11). Accumulating clinical trials have suggested that the combination of ATG and CsA therapy improved the survival of AA patients (31-33). However, 20-40\% of patients present refractory or relapse following IST (10-13).

In the current study, the ORR of patients treated by a combination of IST and UCBI was $81.8 \%$, which was notably higher when compared with that of patients treated only by IST (57.1\%). It was also observed that the rate at which ANC and platelet count responses were achieved in the IST+UCBI group was evidently faster in comparison with that in the IST group. In addition, treatment with IST combined with UCBI was illustrated to be an independent factor able to predict a better ORR in patients, and this advantageous treatment efficacy of the IST+UCBI group may be associated with the UCBI treatment. UCB contains a considerable amount of hematopoietic stem cells (HSCs) and hematopoietic progenitor cells (HPCs), which are the most required hematopoietic cells in AA patients $(1,19)$. HSCs and HPCs have the ability to engraft better than bone marrow, contributing to the restoration of blood cells in AA patients (34-36). In terms of the pathological process, HSCs and HPCs are attacked by the overactivated Tc1, which results in increased apoptosis and the cell cycle arrest of HSCs and HPCs, and ultimately leads to a decreased proliferation of HSCs and HPCs (37-39). Thereby, regulatory T cells (Tregs), naïve T and antigen unexperienced T cells in UCB may serve crucial roles in the immune reconstitution in AA patients (40). Tregs are a subtype of $\mathrm{T}$ cells that are able to inhibit the autoimmune disease by suppressing the activation of the immune system and preventing the pathological immune responses, according to previous findings (41). AA is an immune disorder in which the hematopoietic system is attacked by over activated $\mathrm{T}$ cells, which suggests that Tregs may be involved in the regulation of the immune disorder in patients with AA. CsA used in the IST strategy in the current study, is a type of calcineurin inhibitor, known to inhibit the T-cell differentiation to Treg, indicating 
Table V. Adverse events of patients in the IST and IST+UCBT groups.

\begin{tabular}{lcrr}
\hline Side effects & IST group $(\mathrm{n}=33)$ & IST+UCBI group (n=35) & P-value \\
\hline Fever & $13(39.4)$ & $19(54.3)$ & 0.218 \\
Rush & $10(30.3)$ & $8(22.9)$ & 0.486 \\
ATG-associated serum disease & $9(27.3)$ & $12(34.3)$ & 0.531 \\
Infection & $22(33.3)$ & $21(60)$ & 0.568 \\
Hemorrhage & $15(45.5)$ & $13(37.1)$ & 0.486 \\
\hline
\end{tabular}

Data are presented as the $\mathrm{n}(\%)$. Comparison between groups was evaluated by $\chi^{2}$ test. IST, immunosuppressive therapy; UCBI, umbilical cord blood infusion; ATG, antithymocyte globulin.
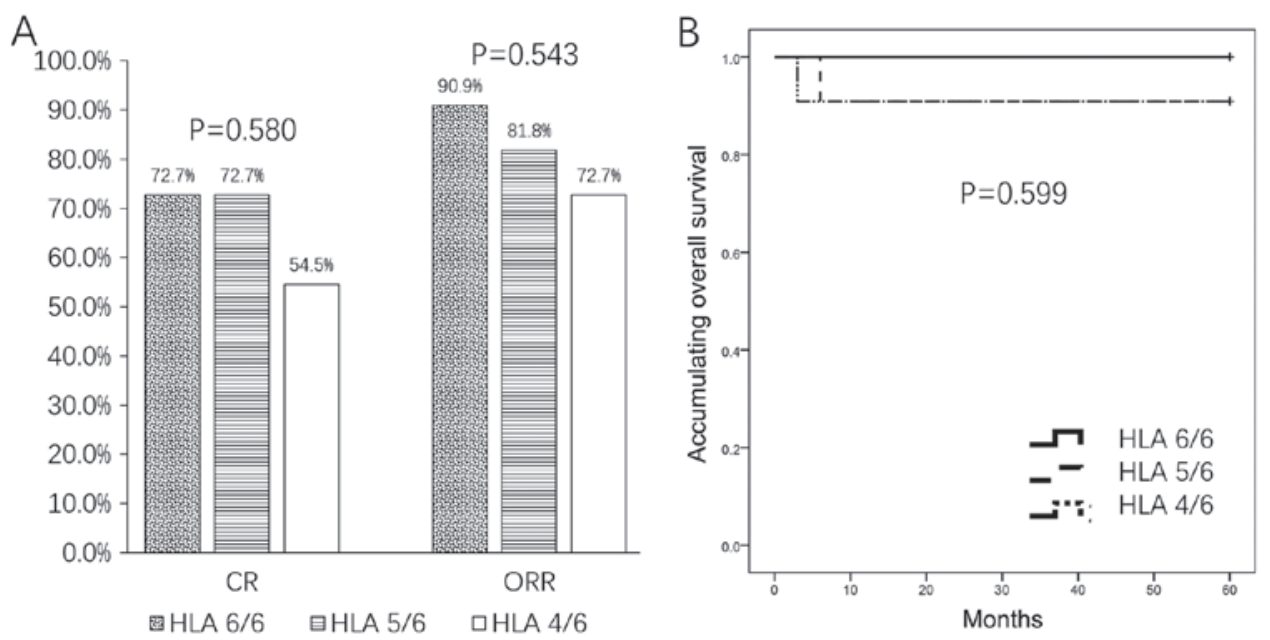

Figure 4. Subgroup analysis in patients with diversified degrees of HLA match. (A) No difference was observed in the CR and ORR in patients with different degrees of HLA match, while (B) the OS was also not significantly altered. $\chi^{2}$ test was performed to evaluate the difference in CR and ORR among the groups. Kaplan-Meier curve was performed to evaluate the OS between groups. Comparisons were analyzed by the log-rank test. HLA, human leukocyte antigen; ORR, overall response rate; $\mathrm{CR}$, complete response.

that CsA may ameliorate the regulation effect of Tregs in AA patients. Although previous studies have reported that CsA inhibits $\mathrm{T}$ cell proliferation and activation, to the best of our knowledge, there is no evidence of CsA inhibiting Tregs in patients with AA. In addition, several previous studies have elucidated that treatment with CsA may increase the number of Tregs in patients with AA and other diseases (42-44). Previous studies have indicated good efficacy of CsA in the treatment of patients with AA, which may indicate that if it existed, the inhibition of Tregs by CsA may be limited and does not interfere with the treatment effect (1,45-47).

UCB has been proven to have reduced immunogenicity compared with bone marrow, which results in more optimal engrafting in recipients (48). In several previous studies, UCBI subsequent to or in combination with IST was observed to be of relatively good efficacy (49-51). In the study by Xie et al (49) UCBI was used subsequent to intensive IST for the treatment of SAA children, resulting in CR and PR of 50.4 and $26.3 \%$, respectively, after 6 months, which was similar to the present study results. UCBI has also been used in adult SAA patients, combined with high-dose IST, and the total effective treatment rate $(\mathrm{CR}+\mathrm{PR})$ of SAA patients was $72 \%$, which was greater compared with that of SAA patients (57.14\%) (50). In addition, another study used a high dose of cyclophosphamide as an
IST strategy combined with UCBI for the treatment of SAA patients and revealed that the recovery times of ANC and platelets were only 23 and 37 days after UCBI, respectively, which was markedly reduced compared with the high-dose cyclophosphamide treatment alone (51). These results of previous studies were in accordance with the findings of the current study.

However, the present study did not detect a difference in the survival of SAA and VSAA patients, which may be due to the following factors: i) The sample size of 68 patients was small; ii) although the infusion of UCB restored the blood cells in a relatively short time, the expansion of HSCs and HPCs in the UCB is limited compared with that of bone marrow; iii) one patient received HSCT following therapy due to a poor response, which resulted in the improvement of survival; and iv) the supportive therapy also contributed to the recovery of blood cells and the prevention of infections, thus improving the survival of VSAA patients. Additionally, a safety assessment demonstrated that UCBI did not increase the adverse events in SAA patients compared with those treated with IST alone.

HLA is essential for assessing the likelihood of transplant rejection, a higher degree of HLA match indicates a reduced probability of transplant rejection (52). In the present study, no difference between clinical response and OS was observed in 
patients with diversified degrees of HLA match. These results may be due to the small sample size, as well as the fact that part of the patients received HSCT or other therapies subsequent to the IST and UCBI treatment, potentially improving their survival to a certain extent.

Various limitations existed in the present study. Firstly, there may be certain compounding factors, including the interval between diagnosis and IST initiation in patients; however, logistic regression was performed to minimize these factors. In addition, the sample size used was relatively small. Furthermore, there may have been crossover between the disease severity of AA and the blood count, as well as $\mathrm{Hb}$ level, in the logistic regression analysis, however, since the blood count and $\mathrm{Hb}$ were continuous variables, while the disease severity was a classified variable, these parameters were not affected by each other.

In conclusion, patients with SAA treated by combined IST plus UCBI were observed to have favorable treatment responses, the rapid recovery of ANC and platelets, as well as a similar frequency of adverse events when compared with patients treated with combined IST only. The results of the present study indicated that combined IST plus UCBI treatment may be applied as an effective and safe therapy for patients with SAA. Additionally, the present study used a relatively small sample size so further studies should enlarge the sample size to evaluate the efficacy and safety of combined IST plus UCBI in patients with SAA.

\section{Acknowledgements}

The present study was supported by the National Natural Science Foundation of China (grant no. 81700101).

\section{References}

1. Young NS, Calado RT and Scheinberg P: Current concepts in the pathophysiology and treatment of aplastic anemia. Blood 108: 2509-2519, 2006.

2. Killick SB, Bown N, Cavenagh J, Dokal I, Foukaneli T, Hill A, Hillmen P, Ireland R, Kulasekararaj A, Mufti G, et al: Guidelines for the diagnosis and management of adult aplastic anaemia. Br J Haematol 172: 187-207, 2016.

3. Young NS: Hematopoietic cell destruction by immune mechanisms in acquired aplastic anemia. Semin Hematol 37: 3-14, 2000.

4. Ishiyama K: Immune pathophysiology of acquired aplastic anemia. Rinsho Ketsueki 57: 525-530, 2016 (In Japanese).

5. Zhang T, Wang J, Zhou X, Liang R, Bai Q, Yang L, Gu H, Gao G, Dong $\mathrm{B}$, Zhu $\mathrm{H}$ and Chen $\mathrm{X}$ : Increased expression of TIGIT on CD4+ T cells ameliorates immune-mediated bone marrow failure of aplastic anemia. J Cell Biochem 115: 1918-1927, 2014.

6. Sun YX, Li H, Feng Q, Li X, Yu YY, Zhou LW, Gao Y, Li GS, Ren J, Ma CH, et al: Dysregulated miR34a/diacylglycerol kinase $\zeta$ interaction enhances T-cell activation in acquired aplastic anemia. Oncotarget 8: 6142-6154, 2017.

7. Zhang J, Gu Y, Xu C and Qu X: Increased T cell immunoglobulin mucin-3 and its ligand in acquired aplastic anemia. Eur J Haematol 81: 130-139, 2008.

8. Scheinberg P and Young NS: How I treat acquired aplastic anemia. Blood 120: 1185-1196, 2012.

9. Miano M and Dufour C: The diagnosis and treatment of aplastic anemia: A review. Int J Hematol 101: 527-535, 2015.

10. Frickhofen N, Heimpel H, Kaltwasser JP and Schrezenmeier H; German Aplastic Anemia Study Group: Antithymocyte globulin with or without cyclosporin A: 11-year follow-up of a randomized trial comparing treatments of aplastic anemia. Blood 101: 1236-1242, 2003.
11. Rosenfeld S, Follmann D, Nunez O and Young NS: Antithymocyte globulin and cyclosporine for severe aplastic anemia: Association between hematologic response and long-term outcome. JAMA 289: 1130-1135, 2003.

12. Marsh JC, Ball SE, Cavenagh J, Darbyshire P, Dokal I, Gordon-Smith EC, Keidan J, Laurie A, Martin A, Mercieca J, et al: Guidelines for the diagnosis and management of aplastic anaemia. Br J Haematol 147: 43-70, 2009.

13. Scheinberg P, Nunez O and Young NS: Retreatment with rabbit anti-thymocyte globulin and ciclosporin for patients with relapsed or refractory severe aplastic anaemia. Br J Haematol 133: 622-627, 2006.

14. Ende N, Chen R and Reddi AS: Effect of human umbilical cord blood cells on glycemia and insulitis in type 1 diabetic mice. Biochem Biophys Res Commun 325: 665-669, 2004.

15. Viener H, Brusko T, Wasserfall C, Mcgrail K, Staba S, Cogle C, Atkinson M, Schatz DA and Haller MJ: Changes in regulatory $\mathrm{T}$ cells following autologous umbillical cord blood transfusion in children with type 1 diabetes. Diabetes 56: A82, 2007.

16. Haller MJ, Wasserfall CH, Hulme MA, Cintron M, Brusko TM, McGrail KM, Wingard JR, Theriaque DW, Shuster JJ, Ferguson RJ, et al: Autologous umbilical cord blood infusion followed by oral docosahexaenoic acid and vitamin D supplementation for C-peptide preservation in children with Type 1 diabetes. Biol Blood Marrow Transplant 19: 1126-1129, 2013.

17. Fruchtman S: Stem cell transplantation. Mt Sinai J Med 70: 166-170, 2003.

18. Han P, Hodge G, Story C and Xu X: Phenotypic analysis of functional T-lymphocyte subtypes and natural killer cells in human cord blood: Relevance to umbilical cord blood transplantation. Br J Haematol 89: 733-740, 1995.

19. Xiao Y, Jiang ZJ, Pang Y, Li L, Gao Y, Xiao HW, Li YH, Zhang $\mathrm{H}$ and Liu Q: Efficacy and safety of mesenchymal stromal cell treatment from related donors for patients with refractory aplastic anemia. Cytotherapy 15: 760-766, 2013.

20. Liu HL, Sun ZM, Geng LQ, Wang XB, Ding KY, Tang BI, Tong J and Wang ZY: Unrelated cord blood transplantation for newly diagnosed patients with severe acquired aplastic anemia using a reduced-intensity conditioning: High graft rejection, but good survival. Bone Marrow Transplant 47: 1186-1190, 2012.

21. Camitta BM, Storb R and Thomas ED: Aplastic anemia (second of two parts): Pathogenesis, diagnosis, treatment, and prognosis. N Engl J Med 306: 712-718, 1982.

22. Camitta BM, Storb R and Thomas ED: Aplastic anemia (first of two parts): Pathogenesis, diagnosis, treatment, and prognosis. N Engl J Med 306: 645-652, 1982.

23. Auerbach AD: Fanconi anemia and its diagnosis. Mutat Res 668: 4-10, 2009.

24. World Medical Association: World medical association declaration of helsinki: Ethical principles for medical research involving human subjects. JAMA 310: 2191-2194, 2013.

25. Bacigalupo A, Hows J, Gluckman E, Nissen C, Marsh J, Van Lint MT, Congiu M, De Planque MM, Ernst P, McCann S, et al: Bone marrow transplantation (BMT) versus immunosuppression for the treatment of severe aplastic anaemia (SAA): A report of the EBMT SAA working party. Br J Haematol 70: 177-182, 1988.

26. Camitta BM, Thomas ED, Nathan DG, Santos G, Gordon-Smith EC, Gale RP, Rappeport JM and Storb R: Severe aplastic anemia: A prospective study of the effect of early marrow transplantation on acute mortality. Blood 48: 63-70, 1976.

27. Kojima S, Hibi S, Kosaka Y, Yamamoto M, Tsuchida M, Mugishima H, Sugita K, Yabe H, Ohara A and Tsukimoto I: Immunosuppressive therapy using antithymocyte globulin, cyclosporine, and danazol with or without human granulocyte colony-stimulating factor in children with acquired aplastic anemia. Blood 96: 2049-2054, 2000.

28. Goldenberg NA, Graham DK, Liang X and Hays T: Successful treatment of severe aplastic anemia in children using standardized immunosuppressive therapy with antithymocyte globulin and cyclosporine A. Pediatr Blood Cancer 43: 718-722, 2004.

29. Solmaz S, Korur A, Yeral M, Gereklioglu C, Ulusan SN, Boga C and Ozdogu H: Active invasive fungal infection in a patient with severe aplastic anemia. Exp Clin Transplant: Apr 7, 2016 (Epub ahead of print).

30. Mohty M: Mechanisms of action of antithymocyte globulin: T-cell depletion and beyond. Leukemia 21: 1387-1394, 2007. 
31. Frickhofen N, Kaltwasser JP, Schrezenmeier H, Raghavachar A Vogt HG, Herrmann F, Freund M, Meusers P, Salama A and Heimpel H: Treatment of aplastic anemia with antilymphocyte globulin and methylprednisolone with or without cyclosporine The German aplastic anemia study group. N Engl J Med 324: 1297-1304, 1991.

32. Rosenfeld SJ, Kimball J, Vining D and Young NS: Intensive immunosuppression with antithymocyte globulin and cyclosporine as treatment for severe acquired aplastic anemia. Blood 85: 3058-3065, 1995.

33. Bacigalupo A, Broccia G, Corda G, Arcese W, Carotenuto M, Gallamini A, Locatelli F, Mori PG, Saracco P, Todeschini G, et al: Antilymphocyte globulin, cyclosporin, and granulocyte colony-stimulating factor in patients with acquired severe aplastic anemia (SAA): A pilot study of the EBMT SAA working party. Blood 85: 1348-1353, 1995.

34. Broxmeyer HE, Lee MR, Hangoc G, Cooper S, Prasain N, Kim YJ, Mallett C, Ye Z, Witting S, Cornetta K, et al: Hematopoietic stem/progenitor cells, generation of induced pluripotent stem cells, and isolation of endothelial progenitors from 21- to 23.5-year cryopreserved cord blood. Blood 117 4773-4777, 2011.

35. Vormoor J, Lapidot T, Pflumio F, Risdon G, Patterson B Broxmeyer HE and Dick JE: Immature human cord blood progenitors engraft and proliferate to high levels in severe combined immunodeficient mice. Blood 83: 2489-2497, 1994.

36. Bock TA, Orlic D, Dunbar CE, Broxmeyer HE and Bodine DM: Improved engraftment of human hematopoietic cells in severe combined immunodeficient (SCID) mice carrying human cytokine transgenes. J Exp Med 182: 2037-2043, 1995.

37. Sloand E, Kim S, Maciejewski JP, Tisdale J, Follmann D and Young NS: Intracellular interferon-gamma in circulating and marrow $\mathrm{T}$ cells detected by flow cytometry and the response to immunosuppressive therapy in patients with aplastic anemia. Blood 100: 1185-1191, 2002

38. Risitano AM, Maciejewski JP, Green S, Plasilova M, Zeng W and Young NS: In-vivo dominant immune responses in aplastic anaemia: Molecular tracking of putatively pathogenetic T-cell clones by TCR beta-CDR3 sequencing. Lancet 364: 355-364, 2004.

39. Maciejewski J, Selleri C, Anderson S and Young NS: Fas antigen expression on CD34+ human marrow cells is induced by interferon gamma and tumor necrosis factor alpha and potentiates cytokine-mediated hematopoietic suppression in vitro. Blood 85: 3183-3190, 1995.

40. Godfrey WR, Spoden DJ, Ge YG, Baker SR, Liu B, Levine BL, June CH, Blazar BR and Porter SB: Cord blood CD4(+) $\mathrm{CD} 25(+)$-derived $\mathrm{T}$ regulatory cell lines express FoxP3 protein and manifest potent suppressor function. Blood 105: 750-758, 2005.

41. Yamaguchi T, Wing JB and Sakaguchi S: Two modes of immune suppression by Foxp3(+) regulatory T cells under inflammatory or non-inflammatory conditions. Semin Immunol 23: 424-430, 2011.

42. Dao AT, Yamazaki H, Takamatsu H, Sugimori C, Katagiri T, Maruyama H, Zaimoku Y, Maruyama K, Ly TQ, Espinoza L and Nakao S: Cyclosporine restores hematopoietic function by compensating for decreased Tregs in patients with pure red cell aplasia and acquired aplastic anemia. Ann Hematol 95: 771-781, 2016.
43. Brandt C, Pavlovic V, Radbruch A, Worm M and Baumgrass R: Low-dose cyclosporine A therapy increases the regulatory $\mathrm{T}$ cell population in patients with atopic dermatitis. Allergy 64: 1588-1596, 2009

44. Denecke C, Reutzel-Selke A, Sawitzki B, Boenisch O, Khalpey Z, Seifert M, Pratschke J, Volk HD and Tullius SG: Low-dose cyclosporine mediates donor hyporesponsiveness in a fully mismatched rat kidney transplant model. Transpl Immunol 26: 176-185, 2012.

45. Li H, Wang L, Pang Y, Jiang Z, Liu Z, Xiao H, Chen H, Ge X, Lan $\mathrm{H}$ and Xiao $\mathrm{Y}$ : In patients with chronic aplastic anemia, bone marrow-derived MSCs regulate the Treg/Th17 balance by influencing the Notch/RBP-J/FOXP3/ROR $\gamma$ t pathway. Sci Rep 7: 42488, 2017.

46. Garg R, Faderl S, Garcia-Manero G, Cortes J, Koller C, Huang X, York S, Pierce S, Brandt M, Beran M, et al: Phase II study of rabbit anti-thymocyte globulin, cyclosporine and granulocyte colony-stimulating factor in patients with aplastic anemia and myelodysplastic syndrome. Leukemia 23: 1297-1302, 2009.

47. Mandal PK, Baul S, Dolai TK, De R and Chakrabarti P: Outcome of cyclosporine monotherapy in patients of aplastic anemia: Experience of a tertiary care hospital in Eastern India. Indian J Hematol Blood Transfus 33: 144-147, 2017.

48. Rocha V, Wagner JE Jr, Sobocinski KA, Klein JP, Zhang MJ, Horowitz MM and Gluckman E: Graft-versus-host disease in children who have received a cord-blood or bone marrow transplant from an HLA-identical sibling. Eurocord and international bone marrow transplant registry working committee on alternative donor and stem cell sources. N Engl J Med 342: 1846-1854, 2000.

49. Xie LN, Fang Y, Yu Z, Song NX, Kong FS, Liu XM and Zhou F: Increased immunosuppressive treatment combined with unrelated umbilical cord blood infusion in children with severe aplastic anemia. Cell Immunol 289: 150-154, 2014.

50. Yu Z, Zhou F, Fu Ge L, Liu XM, Fang Y, Xie L, Kong FS and Song NX: High-dose immunosuppressive therapy combined with cord blood infusion and non-myeloablative peripheral blood stem cell transplantation for patients with severe aplastic anemia. Eur Rev Med Pharmacol Sci 17: 2613-2618, 2013.

51. Li Y, Sheng Z, Niu S, Ge L, Ren C and Zou Y: Rapid and complete reconstitution of autologous haemopoiesis after cord blood infusion in treatment-naive patients with severe aplastic anemia receiving high-dose cyclophosphamide/ATG therapy. Eur J Haematol 90: 45-50, 2013.

52. Zhang $X$ and Reinsmoen NL: Impact of non-human leukocyte antigen-specific antibodies in kidney and heart transplantation. Front Immunol 8: 434, 2017.

This work is licensed under a Creative Commons Attribution-NonCommercial-NoDerivatives 4.0 International (CC BY-NC-ND 4.0) License. 